\title{
SISTEMA DE AQUISIÇÃO DE DADOS PARA A MÁQUINA DE IMPACTO CHARPY
}

\author{
J. L. MORAES, E. CAVALCANTI NETO, R. BESSA, J. A. BESSA, L. BRAGA e A. R. ALEXANDRIA \\ Laboratório Ensaios Mecânicos (LEM) - Instituto Federal do Ceará \\ auzuir@gmail.com
}

Artigo submetido em fevereiro/2014 e aceito em agosto/2014

DOI: $10.15628 /$ holos.2014.2008

\section{RESUMO}

Este trabalho tem o objetivo de implementar e desenvolver um sistema de aquisição de dados para a máquina de impacto Charpy. Assim, é realizado um estudo da máquina de impacto Charpy, do ensaio de impacto e das ferramentas necessárias para desenvolvimento do projeto. Utiliza-se um acelerômetro para determinar a aceleração nos eixos $x$ e y do pêndulo Charpy durante a realização do ensaio. Para leitura e interpretação dos dados enviados pelo acelerômetro utiliza-se a plataforma de hardware Arduino UNO com software específico. Os dados enviados ao Arduino são apresentados em uma interface gráfica desenvolvida no
Matlab. Nesta interface é possível inserir os dados iniciais de ensaio Charpy e apresentar ao usuário final os resultados finais de ensaio, como a energia de impacto, a resistência de impacto e a força necessária para romper o corpo de prova. Além disso, é apresentado ao usuário um gráfico da aceleração ao longo da realização do ensaio e o gráfico de força ao longo do tempo. Desta forma, registram-se os dados em um arquivo específico para análise e estudo posterior. A porcentagem de erro entre o valor medidor no mostrador da máquina e o resultado automatizado não ultrapassa o limite de $8 \%$.

PALAVRAS-CHAVE: Ensaio Charpy, instrumentação, automação e sensores eletrônicos.

\section{DATA ACQUISITION SYSTEM FOR CHARPY IMPACT MACHINE}

\begin{abstract}
This work is aimed to develop and implement a data acquisition system for the Charpy impact machine. Thus, a study of the Charpy machine, the impact test and the necessary tools to perform the project is performed. It is used an accelerometer to determine the acceleration in the $x$ and $y$ axes of the pendulum during Charpy testing. To read the data sent by the accelerometer is used the hardware platform Arduino UNO with specific software. The data sent by Arduino was acquired by an interface developed on Matlab using a graphic interface. On this
\end{abstract}

interface is possible to insert initial data from the Charpy test and shows to the user the final result from the test, such as impact energy, impact resistance, needed power to break the specimen. Beyond that, it is shown to the user the graphic of acceleration through time on testing and the graphic of power. Therefore, it is registered the data on a specific file for further analysis. The percentage of mistake between the meter value in the display of machine and automated result does not exceeds to the limit of $8 \%$.

KEYWORDS: Charpy testing, instrumentation, automation, electronics sensors. 


\section{INTRODUÇÃO}

O ensaio mais habitual e rudimentar para caracterizar o comportamento mecânico dos materiais em cargas dinâmicas é o ensaio de impacto Charpy (LUCENA E MARTINS, 2006).

O ensaio de impacto é utilizado para avaliar a tenacidade ao impacto de um determinado material e mensurar a energia necessária para fraturar o corpo de prova (COLPAERT, 2008).

As principais vantagens do ensaio Charpy são a simplicidade, a facilidade do ensaio e o baixo custo com a execução do mesmo. No entanto, nota-se que as informações obtidas com a realização do ensaio Charpy convencional são poucas e imprecisas. Com isso, a instrumentação da máquina Charpy disponibiliza um acervo maior de informações, aumentando a confiabilidade dos resultados.

Existem máquinas Charpy instrumentadas em escala comercial, sendo todas importadas e de custo elevado. Não há nenhuma empresa ou instituição que execute a instrumentação de pêndulos Charpy disponibilizando com baixo custo às informações de um ensaio instrumentado (LUCENA E MARTINS, 2006).

Para a instrumentação da máquina de impacto Charpy são utilizados sensores eletrônicos com plataformas específicas e rotinas programáveis de desenvolvimento para cálculo de aceleração, força, energia e resistência, sendo possível traçar gráficos de aceleração e força ao longo do tempo. Tudo isso, em uma interface gráfica de desenvolvimento para registro dos dados e resultados obtidos com a realização do ensaio Charpy. Logo, torna-se possível realizar um estudo comparativo posterior das características mecânicas do material ensaiado.

Percebe-se que a correta instrumentação da máquina de impacto Charpy e a automação do processo de ensaio de impacto permite um aumento do número de informações coletadas no ensaio, elevando consideravelmente a precisão, a confiabilidade e a segurança dos resultados obtidos.

\section{ENSAIO DE IMPACTO CHARPY}

Realizar um ensaio de impacto consiste em submeter um objeto ou um material a situações que simulam os esforços que eles vão sofrer nas condições reais de uso, chegando aos seus limites extremos (COSTA, 2000).

O ensaio de impacto é um ensaio dinâmico e bastante utilizado na Engenharia de Ciências dos Materiais devido à sua dinâmica, simplicidade e rapidez. Este ensaio é aplicado principalmente para materiais em baixa temperatura, como teste de aceitação do material. A tendência de um material de se comportar de uma maneira frágil é medida pelo ensaio de impacto (SOUZA, 1982).

O objetivo principal deste ensaio é medir a quantidade de energia absorvida pelo material durante a fratura, avaliando a sua tenacidade ao impacto (COLPAERT, 2008).

O ensaio de impacto Charpy é utilizado para a estimação de metais, especialmente de aço e de aço fundido, com respeito ao comportamento em relação à solicitação de impacto. Este pode ser utilizado para controle de tratamento a quente e também para a comprovação se existe uma 
tendência de ruptura por separação. Esta permite conclusões a cerca da fragilidade a frio, a fragilidade a quente, envelhecimentos e outras falhas do material (ZWINGEL, 1968).

O ensaio fornece poucas informações e resultados de caráter comparativo por utilizar uma pequena parte da estrutura para análise. Desta forma, as informações são apenas uma indicação qualitativa a respeito do comportamento dúctil-frágil dos materiais (TÓTH, ROSSMANITH, e SIEWERT, 2002).

\subsection{ENSAIO DE IMPACTO CHARPY CONVENCIONAL}

A máquina para a realização do ensaio de Charpy é bastante rudimentar (CALLISTER, 2008).

A máquina Charpy utiliza-se de um mecanismo pendular para ensaio de resistência ao impacto. Esta é constituída de uma armação, do pêndulo, da fixação do corpo de prova e do medidor analógico (ZWINGEL, 1968).

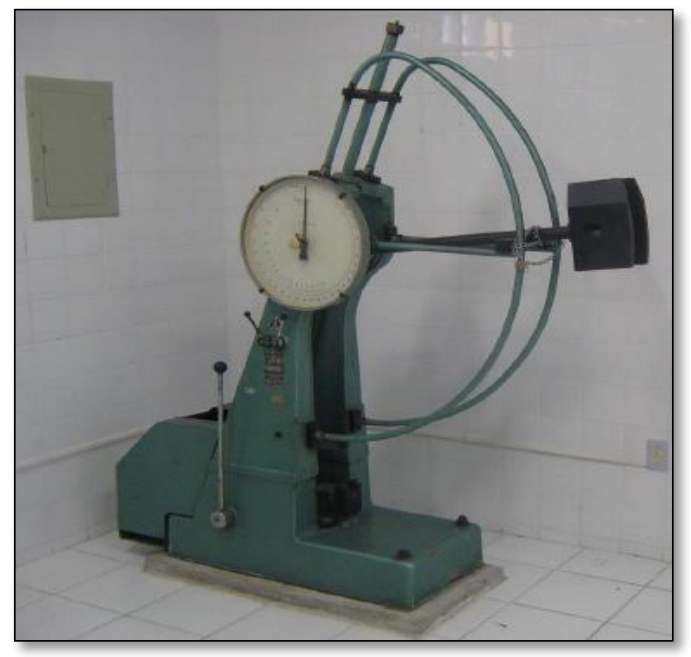

Figura 1-Máquina de impacto Charpy instalada no IFCE no laboratório de ensaios mecânicos (LEM).

A máquina de ensaio de impacto Charpy, tema deste projeto, está instalada no Instituto Federal do Ceará (IFCE) no Laboratório de Ensaios Mecânicos (LEM) e é apresentada na Figura 1.

Os corpos de prova para o ensaio Charpy são do tipo entalhado e são especificados pela norma americana E-23 ASTM. Estes se dividem em três tipos, conforme a forma e o tamanho de seu entalhe, sendo esses do tipo A, B e C, e tendo todos eles uma seção quadrada de $10 \mathrm{~mm}$ de lado e um comprimento de $55 \mathrm{~mm}$ (SOUZA, 1982).

$\mathrm{O}$ entalhe do corpo de prova é feito no meio do mesmo. $\mathrm{O}$ do tipo $\mathrm{A}$ em forma de um $\mathrm{V}$, o do tipo $B$ em forma de uma fechadura e do tipo $C$ em forma de um $U$ invertido (DALCIN, 2007). Os tipos de corpo de prova podem ser visualizados na Figura 2. 


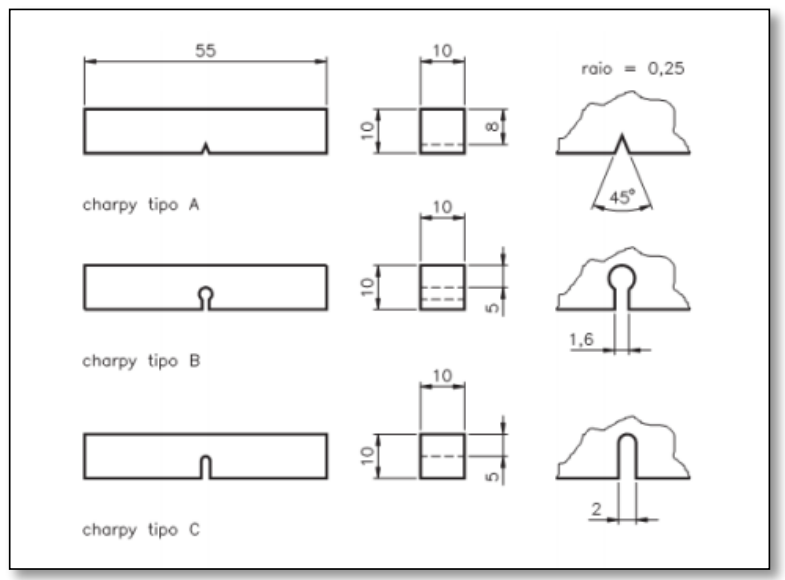

Figura 2-Tipos de corpo de prova do ensaio de impacto Charpy. Fonte: (DALCIN, 2007).

Pela análise da Figura 2, pode-se perceber que os diferentes tipos de corpo de prova, devem possui o mesmo comprimento e a mesma área de seção transversal, alterando apenas o tipo de entalhe.

Para a realização do ensaio, deve-se escolher o tipo mais adequado do entalhe do corpo de prova. Os entalhes mais agudos ou mais profundos são utilizados nos ensaios de caráter mais dúcteis ou com velocidades menores de ensaio.

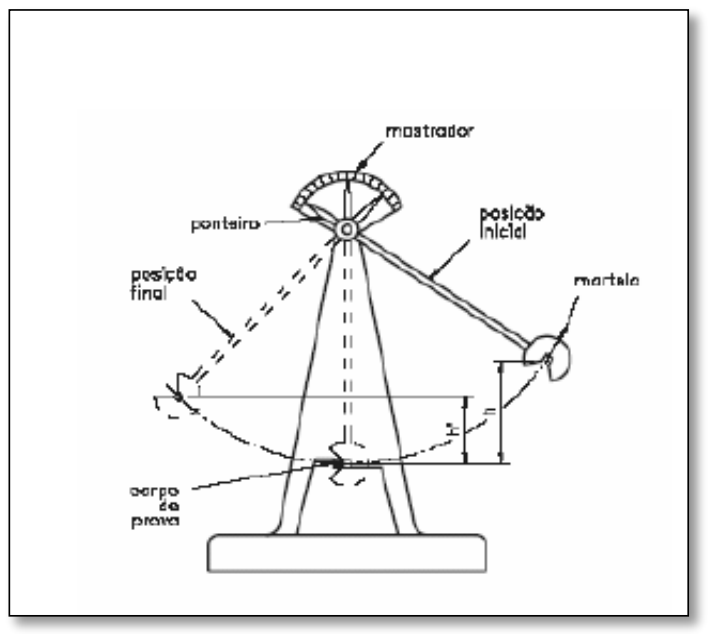

Figura 3 - Máquina de ensaio Charpy e seus componentes. Fonte: (MARTINS e LUCENA, 2006).

A estrutura da máquina de impacto Charpy e os elementos da máquina Charpy e as posições do martelo Charpy durante o período de realização do ensaio são apresentadas na Figura 3.

A técnica de ensaio de impacto Charpy é destrutiva, pois se caracteriza por submeter ao corpo ensaiado uma força brusca e repentina, que deve rompê-lo (DALCIN, 2007).

O método mais comum de ensaio de impacto Charpy é o golpe desferido por um pêndulo em oscilação. O martelo acoplado ao pêndulo é elevado a certa altura, onde o mesmo adquire sua energia potencial inicial, sendo esta fixa e específica. Ao atingir a energia potencial inicial, o martelo é solto e alcança o corpo de prova. Ao atingir o corpo de prova, o martelo sobe a uma 
altura que é inversamente proporcional à energia absorvida para romper o corpo de prova. Sendo assim, quanto maior a altura atingida pelo martelo ao romper o corpo de prova, menor será a energia necessária para fraturar ou rompê-lo (SOUZA, 1988).

A posição atingida pelo martelo ao romper o corpo de prova corresponde à sua energia final. A energia necessária para fraturar o corpo de prova é a diferença entre a energia potencial inicial e final do ensaio de impacto. Esta energia é lida diretamente na máquina, sendo esta apresentada no mostrador/medidor da máquina de impacto Charpy.

O ensaio de impacto Charpy convencional, ou seja, não instrumentado, não é considerado um ensaio muito preciso e eficaz e por isso é recomendado se efetuar o ensaio de impacto mais de uma vez, pois se podem obter resultados diferentes para o mesmo tipo de corpo de prova. Assim, é necessário realizar no mínimo três ensaios para se obter uma média aceitável como resultado (SOUZA, 1982).

O resultado esperado com a realização do ensaio de impacto Charpy é a energia necessária para romper o corpo de prova. Esta energia de impacto é calculada pela diferença entre a energia potencial inicial e a energia potencial final. Pode-se calcular a energia potencial inicial e final pelas equações 1 e 2 , respectivamente.

$$
\begin{aligned}
& E p_{\text {inicial }}=m_{\text {inicial }} \cdot g_{\text {inicial }} \cdot \mathbf{h}_{\text {inicial }} \\
& E p_{\text {ifinal }}=m_{\text {final }} \cdot g_{\text {final }} \cdot \mathbf{h}_{\text {final }}
\end{aligned}
$$

equação (1)

equação (2)

Analisando as equações, tem-se $m_{\text {inicial }}$ e $m_{\text {final }}$ como a massa do martelo e estas não se alteram, pois são grandezas constantes e conhecidas. $g_{\text {inicial }}$ e $g_{\text {final }}$ correspondem a aceleração da gravidade inicial e final, respectivamente, grandezas que também são constantes e conhecidas, e $h_{\text {inicial }}$ e $h_{\text {final }}$ apresentam a altura inicial e final do pêndulo Charpy, respectivamente. A altura inicial do pêndulo é conhecida, no entanto, a altura final é a única variável desconhecida e esta deve ser obtida pela realização do ensaio Charpy. Para determinar a energia necessária para romper o corpo de prova, basta determinar a altura final de ensaio.

\subsection{ENSAIO DE IMPACTO CHARPY INSTRUMENTADO}

A instrumentação da máquina de impacto Charpy tem sido tema de estudo nas últimas décadas. Existem alguns trabalhos que relatam o empenho de pesquisadores em munir a máquina Charpy de sensores eletrônicos, com o objetivo de melhorar a coleta de dados. $O$ ensaio convencional, sem sensores, é caracterizado pela simplicidade do funcionamento e pela conservação da energia mecânica. No entanto, possui uma relativa limitação dos resultados quantitativos medidos com a realização do ensaio.

Segundo Figueiredo (2004), com a instrumentação da máquina de impacto Charpy, podese fazer a análise dos esforços envolvidos durante o impacto através da curva força $x$ tempo.

Com a correta automação da máquina, torna-se possível calcular a aceleração do martelo na execução do ensaio e com isso calcular a força necessária para fraturar o corpo de prova, além da energia e da resistência ao impacto. É possível identificar a variação da força ao longo da realização do ensaio de impacto e com isso determinar características mecânicas específicas de 
diferentes materiais. É possível também a realização de um estudo comparativo com um corpo de prova do mesmo material, submetido ao ensaio em temperaturas diferentes.

Desta forma, pode-se perceber que o ensaio realizado na máquina Charpy torna-se mais proveitoso quando existe uma instrumentação adicional aplicada à máquina, permitindo o aumento do número de informações a cerca do ensaio.

A automação da máquina de impacto Charpy apresenta-se de grande importância para a Engenharia e Ciência dos Materiais e para a área de automação, tendo em vista que ambas as áreas apresentam benefícios com a implementação deste projeto.

A vantagem da automatização do processo está no domínio da tecnologia que permite maior controle do processo e integração com outros sistemas, gerando novos trabalhos (MORAES, 2010).

Para a automação da máquina são necessárias a instalação de sensores eletrônicos e a implementação de rotinas que permitam a automação do processo, além da utilização e implementação de um hardware e um software específico.

\subsection{INSTRUMENTAÇÃO}

Para a automação da máquina de impacto Charpy é efetuada a instalação de sensores eletrônicos na máquina, como também o desenvolvimento de uma plataforma específica de hardware utilizando o Arduino UNO e o acelerômetro como base de automação para o projeto.

\subsubsection{ACELERÔMETRO}

Sensores são dispositivos que captam a energia ao seu redor, podendo esta energia ser luminosa, térmica e cinética. Desta forma, o sensor relaciona essas informações ao tipo de grandeza que precisa ser medido, como: temperatura, pressão, velocidade e aceleração (THOMAZINI e ALBUQUERQUE, 2011).

O sensor utilizado neste projeto é o acelerômetro MMA7260 que é ilustrado na Figura 4.

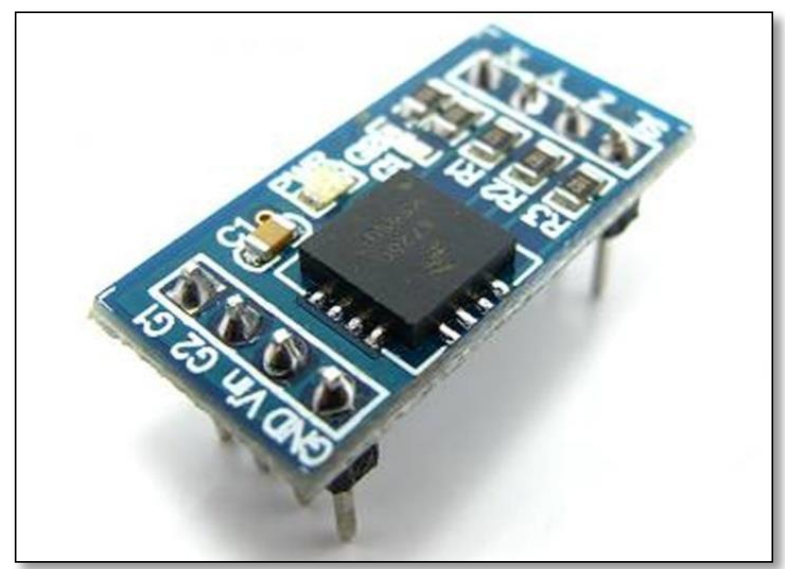

Figura 4- Acelerômetro MMA 7260 Fonte: (Freescale Semiconductor, 2005). 
O acelerômetro MMA 7260 é classificado como um sensor analógico. Estes sensores podem assumir qualquer valor no seu sinal de saída ao longo do tempo, desde que os valores estejam na sua faixa de operação (THOMAZINI e ALBUQUERQUE, 2011).

O acelerômetro MMA7260 é implementado utilizando-se um sistema composto por dois capacitores. Ao analisar a Figura 5, pode-se verificar que as duas placas externas são fixas, e a placa interna é móvel. Quando a massa está em repouso, a distância entre as placas é a mesma, então ambos os capacitores possuem a mesma capacitância. Quando o sistema acelera, a capacitância de cada capacitor varia e com essa variação das capacitâncias é possível determinar um sinal elétrico proporcional à aceleração do sistema. Quando ocorrer uma desestabilização do acelerômetro, esta distância entre as placas irá variar, com isso muda-se a capacitância e, consequentemente, a aceleração do sistema (SANTOS, 2003).

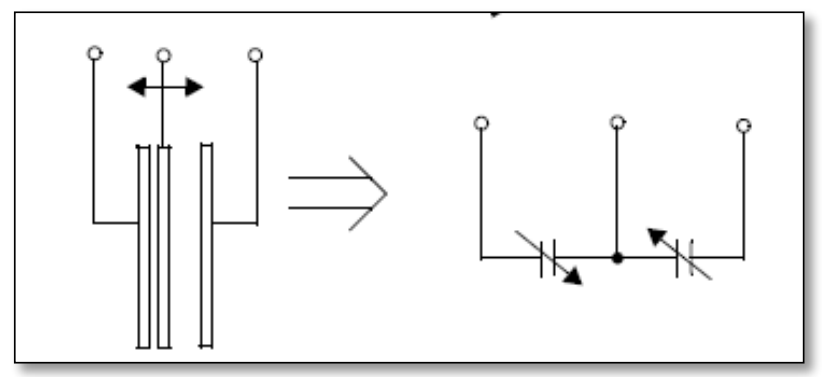

Figura 5 - Acelerômetro MMA 7260. Fonte (Freescale Semiconductor, 2005).

\subsubsection{ARDUINO}

O Arduino é considerado uma plataforma de computação física, baseado em uma placa interligada a um microcontrolador, constituída de Entradas/Saídas (digitais ou analógicas) microcontroladas e desenvolvidas sobre uma biblioteca que facilita a escrita de programação, utilizando com base a linguagem C++ (GUIMARÃES et al, 2010).

O Arduino é um projeto Open-Source que engloba tanto a parte de hardware, como o software, e tem como objetivo fornecer uma plataforma para prototipação de projetos interativos, utilizando um microcontralador (JUSTEN, 2009).

O Arduino consiste de um microcontrolador de placa única e de um software para programá-lo. O hardware é composto de um processador Atmel AVR, um cristal oscilador e um regulador linear de 5 Volts. A placa contém os pinos de entrada e saída em um encaixe padrão para facilitar a conexão de circuitos externos que agregam novas funcionalidades. O software trata-se de uma interface de programação para desenvolvimento do firmware do microcontrolador e do gerenciador de inicialização (bootloader) que é executado na própria placa do Arduino (GOMES e TAVARES, 2013).

Segundo McRoberts (2010), o Arduino é considerado um pequeno computador que pode ser programado para processar entradas e saídas entre dispositivos e os componentes externos ligados a ele. Esse é considerado uma plataforma de computação física e embarcada, ou seja, um sistema que pode interagir com o seu ambiente por meio de hardware e software. O Arduino pode ser utilizado para desenvolver aplicações autônomas, ou pode ser conectado a um computador através da topologia peer-to-peer ou realizar conexões através da rede Internet. Este projeto utiliza 
o Arduino UNO, que por sua vez utiliza o microcontrolador ATMEGA328P, que pode ser observado na Figura 6.

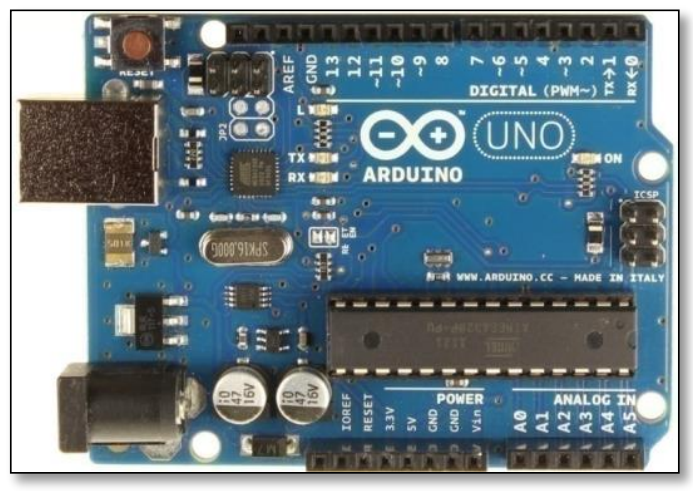

Figura 6 - Arduino UNO. Fonte: (www.arduino.cc/en/Main/ArduinoBoardUno).

\subsubsection{Matlab}

O Matlab utilizado neste projeto é a versão 7.6.0.324 do ano 2008. Este é o software desenvolvido e dedicado a vários tipos de interfaces de programação.

Este software foi utilizado como plataforma de comunicação entre o Arduino, o acelerômetro MMA7260, o computador e o usuário final. No Matlab é desenvolvida uma interface Homem Máquina apta ao usuário final determinar as considerações iniciais de ensaio, realizar o ensaio e visualizar os resultados finais.

\section{MATERIAIS E MÉTODOS}

O ensaio Charpy é realizado para mensurar a energia absorvida após a ruptura do corpo de prova. Para medição desta energia, precisa-se calcular a energia potencial final de ensaio e correlacioná-la com a energia de impacto apresentada no mostrador analógico da máquina.

Para a automação da máquina utilizou um acelerômetro analógico MMA7260 que foi instalado no pêndulo Charpy. $O$ acelerômetro determina os valores de aceleração nos eixos $x$ e $y$ do pêndulo Charpy durante a realização do ensaio.

Percebe-se a grande vantagem e a justificativa da utilização do acelerômetro para este projeto. Com apenas um sensor, pode-se determinar todas as variáveis necessárias para a automação do processo.

Com a utilização do acelerômetro é possível determinar as variáveis necessárias para a automação do ensaio Charpy. No entanto, este necessita de uma plataforma de hardware e software para a leitura, cálculo e interpretação dos dados.

Para a plataforma de hardware e software utilizou-se o Arduino UNO. Este possui um microcontrolador ATMEGA 238P, entradas e saídas analógicas e digitais, memória EEPROM e um programador específico com linguagem de programação específica baseada em linguagem $C / C++$. 
O acelerômetro foi interligado ao Arduino através das entradas analógicas e com isso foi possível através de uma lógica de programação executada no próprio Arduino, ler os dados enviados pelo acelerômetro.

O acelerômetro envia os dados para o Arduino e este é responsável por receber estes dados e efetuar os cálculos e lógicas necessárias para obtenção dos resultados finais do ensaio de impacto Charpy. O Matlab recebe os dados do Arduino e na interface gráfica é possível especificar usuário, tipo de material ensaiado, temperatura do corpo de prova, entre outros. Como também gerar gráficos da simulação do ensaio Charpy, gráfico de Força x Tempo e registrar os resultados obtidos com a realização do ensaio de impacto.

Realizou-se um ensaio Charpy e foi efetuada a leitura dos eixos $x$ e $y$ através do Arduino e visualizado os dados no Matlab em forma gráfica. Com os dados salvos torna-se possível realizar um estudo comportamental dos eixos de aceleração e desta forma determinar as variáveis do processo.

Na Figura 7 pode-se observar a oscilação dos eixos de aceleração durante a realização do ensaio de impacto.

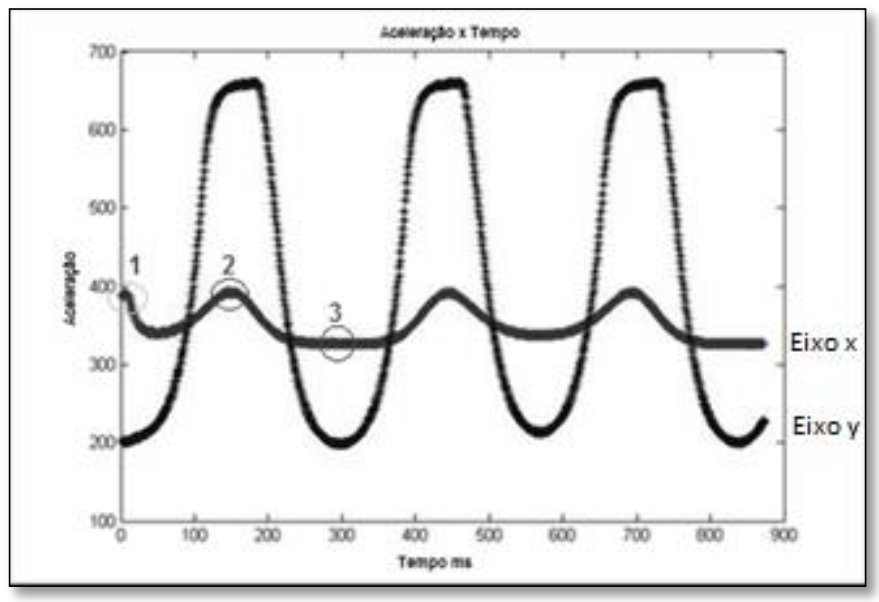

Figura 6 - Leitura dos Eixos de Aceleração x e y.

Na Figura 7 pode-se analisar como os eixos $x$ e $y$ se comportam durante a oscilação do pêndulo Charpy. O ponto 1 equivale ao início do ensaio Charpy com o pêndulo na sua posição inicial, o ponto 2 equivale ao momento que o pêndulo cruza o suporte do corpo de prova, ou seja, rompimento do corpo de prova e o ponto 3 equivale ao momento que o pêndulo atinge a sua altura máxima de ensaio e retorna com o movimento oscilatório decrescente.

Pela Figura 7 pode-se analisar que o valor máximo de aceleração corresponde ao instante de ruptura do corpo de prova e o valor mínimo de aceleração corresponde a altura máxima do pêndulo Charpy. Com a determinação da altura máxima final de ensaio, pode-se assim calcular a energia de impacto final de ensaio. Tendo em vista que a massa do martelo e a aceleração da gravidade são constantes, a única variável desconhecida é a altura máxima de ensaio.

Para cálculo da energia de impacto se faz necessário definir a função aproximada de relação entre a energia final de ensaio e o resultado do mostrador analógico da máquina. 
Para isso é necessário calcular duas vezes a integral trapezoidal dos valores de aceleração ao longo tempo. Com a primeira integral trapezoidal determinam-se os valores de velocidade ao longo do tempo. Com a segunda integral trapezoidal definem-se os valores de deslocamento linear do pêndulo Charpy. O cálculo dessas duas integrais chama-se de integral dupla. Calcula-se a integral trapezoidal pela equação 3.

$$
\sum_{\text {impacto }}=\int_{\mathbf{a}}^{\mathbf{b}} \mathbf{f}(\mathbf{x}) \mathbf{d x}=(\mathbf{b}-\mathbf{a})\left\{\frac{\mathbf{f}(\mathbf{a})+\mathbf{f}(\mathbf{b})}{2}\right\}
$$

A integral trapezoidal procede de quatro etapas principais que é a decomposição do domínio em pedaços ao longo do tempo, a integração aproximada de cada pedaço, da soma dos resultados numéricos obtidos e da média da soma dos resultados. Com o cálculo duplo da integral da aceleração é possível determinar o deslocamento do pêndulo e com isso constatar o ponto 3 da Figura 7.

Define-se o ponto 3 da Figura 7 e realiza um estudo da relação entre o ponto mínimo de deslocamento com o resultado de impacto apresentado no mostrador da máquina de impacto Charpy.

A dupla integração trapezoidal permite definir o deslocamento do pêndulo Charpy, como também determinar o ponto mínimo de deslocamento. Após a leitura dos eixos de aceleração e cálculo de integração dupla realizada pelo Matlab é possível traçar o gráfico de deslocamento ao longo do tempo. O gráfico de deslocamento pelo tempo pode ser analisado na Figura 8.

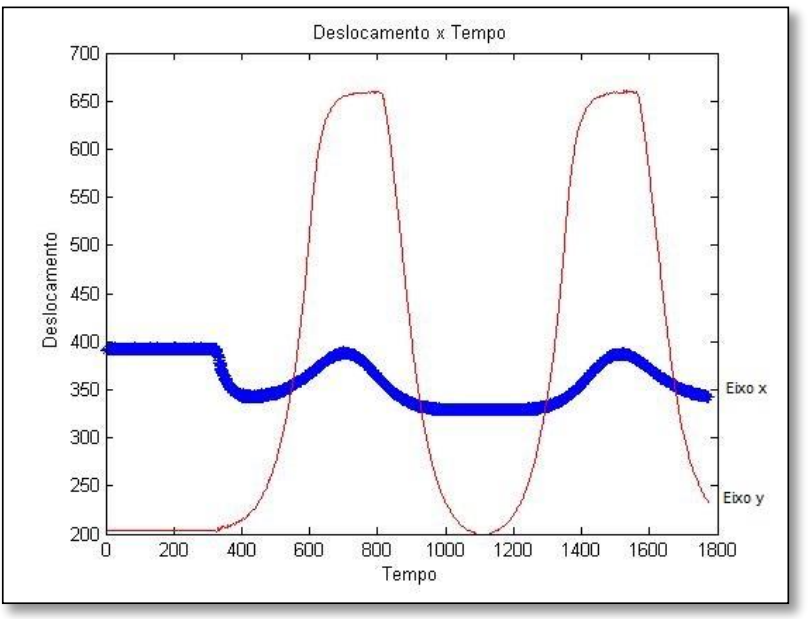

Figura 8 - Gráfico do deslocamento durante a realização do ensaio Charpy sem carga.

A Figura 8 corresponde a um ensaio de impacto Charpy sem carga e ao analisar esta figura pode-se perceber que o comportamento dos eixos é semelhante ao gráfico de aceleração pelo tempo e que o ponto mínimo de deslocamento corresponde ao instante que o pêndulo atinge a sua altura máxima após romper o corpo de prova. Nesse instante é possível determinar a energia de impacto. No entanto, os eixos de aceleração possuem comportamento distinto quando a máquina é submetida a uma carga. Pode-se perceber a alteração comportamental dos eixos de aceleração pela Figura 9 com o gráfico de deslocamento durante a realização do ensaio de impacto Charpy com carga. 


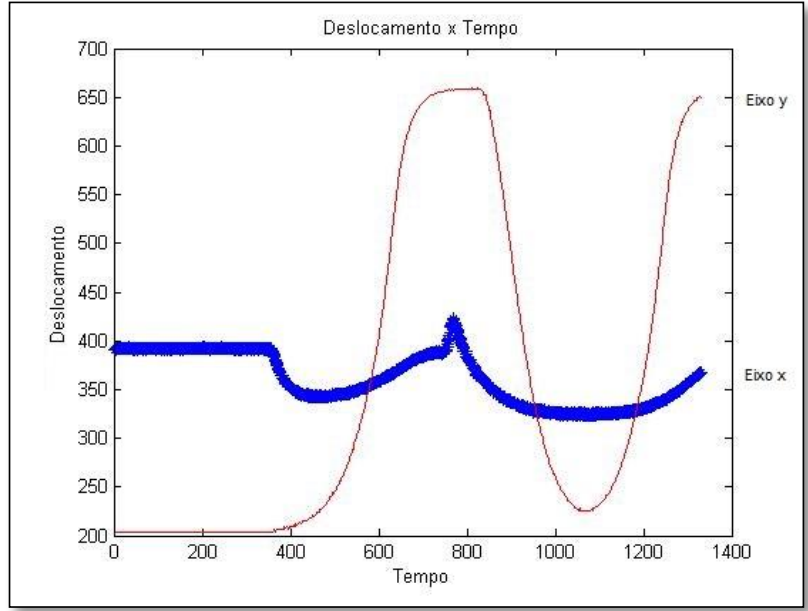

Figura 9 - Gráfico do deslocamento durante a realização do ensaio Charpy com carga.

Pela Figura 9 pode-se analisar que apenas o eixo $x$ altera significativamente $o$ seu comportamento e o eixo y permanece com a mesma variação com e sem carga. Logo, para cálculo da energia de impacto apenas o eixo $x$ é necessário. Ao analisar o eixo $x$ pode-se perceber que $o$ momento de alteração do ciclo do gráfico corresponde ao instante de ruptura do corpo de prova. Com isso, as conclusões iniciais são verdadeiras, no ponto máximo de deslocamento ou aceleração é o instante de ruptura do corpo de prova e o ponto mínimo de deslocamento ou aceleração corresponde ao instante de altura máxima do pêndulo e energia potencial final. Na Figura 10 observa-se o gráfico de deslocamento pelo tempo com a determinação do ponto mínimo de deslocamento do eixo $x$.

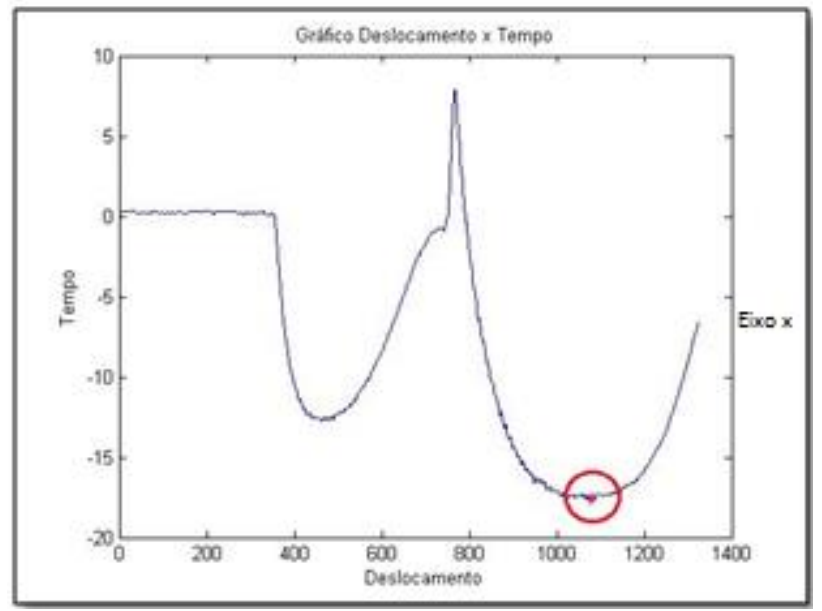

Figura 10 - Gráfico da determinação ponto mínimo de deslocamento.

Na programação do Matlab foi inserida a função para determinação do ponto mínimo de deslocamento, logo, a altura máxima de ensaio e energia final de ensaio. Com isso, pode-se realizar um estudo comparativo dos resultados de energia final automatizada com os resultados do mostrador analógico e determinar da função para cálculo da energia de impacto. O círculo da Figura 10 corresponde ao ponto de deslocamento que determina a altura máxima de ensaio. A energia final automatizada corresponde no cálculo da função polinominal P com a energia final de 
impacto. A função polinominal P calculada pelo Matlab para determinar a relação entre a energia da máquina e a energia automatizada por ser determinada pela equação 4.

$P=1203,19 x^{2}+0,86 x+0,00016$

equação (4)

Foram realizados vários ensaios Charpy com cargas diferentes para determinação da função para cálculo da energia de impacto. Na Tabela 1 apresentam-se os resultados dos ensaios realizados.

Tabela 1 - Resultados dos ensaios Charpy para determinação da energia de impacto.

\begin{tabular}{|c|c|c|c|}
\hline Ensaio & $\begin{array}{c}\text { Energia Máquina } \\
\text { em kpm }\end{array}$ & $\begin{array}{c}\text { Energia Máquina } \\
\text { em Joules }\end{array}$ & $\begin{array}{c}\text { Energia Final } \\
\text { Automatizada }\end{array}$ \\
\hline 1 & 2,60 & 25,49 & $3.280,20$ \\
\hline 2 & 1,70 & 16,67 & $3.226,30$ \\
\hline 3 & 1,30 & 12,74 & $3.183,90$ \\
\hline 4 & 0,52 & 5,09 & $3.083,90$ \\
\hline 5 & 2,01 & 19,71 & $3.202,60$ \\
\hline 6 & 2,82 & 27,65 & $3.333,00$ \\
\hline 7 & 0 & 0 & $3.064,90$ \\
\hline 8 & 4,15 & 40,69 & $3.396,10$ \\
\hline 9 & 5,10 & 50,01 & $3.424,00$ \\
\hline 10 & 5,59 & 54,81 & $3.443,10$ \\
\hline 11 & 3,72 & 36,48 & $3.416,00$ \\
\hline 12 & 3,49 & 34,22 & $3.342,00$ \\
\hline 13 & 2,40 & 23,53 & $3.268,10$ \\
\hline 14 & 3,80 & 37,26 & $3.392,70$ \\
\hline 15 & 5,70 & 55,89 & $3.444,40$ \\
\hline
\end{tabular}

Pela Tabela 1, pode-se analisar que com os resultados de energia da máquina e energia final automatizada foi possível determinar a função para cálculo da energia de impacto. Para isso foi inserido os valores de energia máquina e energia automatizada no Matlab e calculado a função polinominal dessas variáveis. Na Figura 11 analisa-se o gráfico da função da energia de impacto.

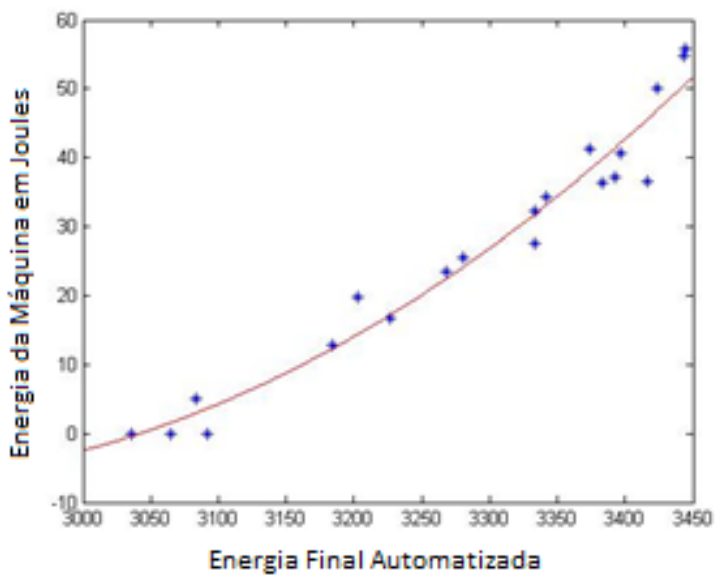

Figura 11 - Gráfico da função da energia de impacto. 
Os pontos dos gráficos correspondem a relação entre a energia da máquina e a energia automatizada e curva correspondem a função polinominal aproximada. Com a determinação desta função é possível calcular a energia de impacto, como também a resistência.

A aceleração equivalente pode ser calculada em todos os pontos de medição de aceleração de $x$ e $y$. Como também se pode calcular a aceleração equivalente total do ensaio através do ponto de aceleração máxima. $O$ cálculo da aceleração equivalente é baseado no triângulo das acelerações que pode ser determinada pela equação 5 .

$$
A c c_{e q u}=\sqrt[2]{a c c_{-} x^{2}}+\sqrt[2]{a c c_{-} y^{2}}
$$

equação (5)

Determinando a aceleração equivalente de ensaio, pode-se calcular a força necessária para romper o corpo de prova.

Após a determinação da aceleração equivalente, o cálculo da força torna-se mais acessível, como a massa do pêndulo é constante, a única variável faltante é a aceleração equivalente. 0 cálculo da força é determinado pela equação 6.

$$
\mathbf{F}=\mathbf{m} \cdot \mathbf{a}
$$

equação (6)

Em que $m$ corresponde à massa do pêndulo e $a$ corresponde à aceleração equivalente. Com a determinação da força torna-se possível traçar um gráfico de força $x$ tempo e com isso definir a força máxima para ruptura do corpo de prova.

\section{RESULTADOS}

Foram realizados testes com os corpos de prova do tipo Latão e Aço $1020 \mathrm{com}$ a interface gráfica. Na tela inicial apresentada Figura 12, todos os dados iniciais de ensaio foram inseridos. Os dados inseridos nesta tela são fundamentais para o estudo posterior do ensaio.

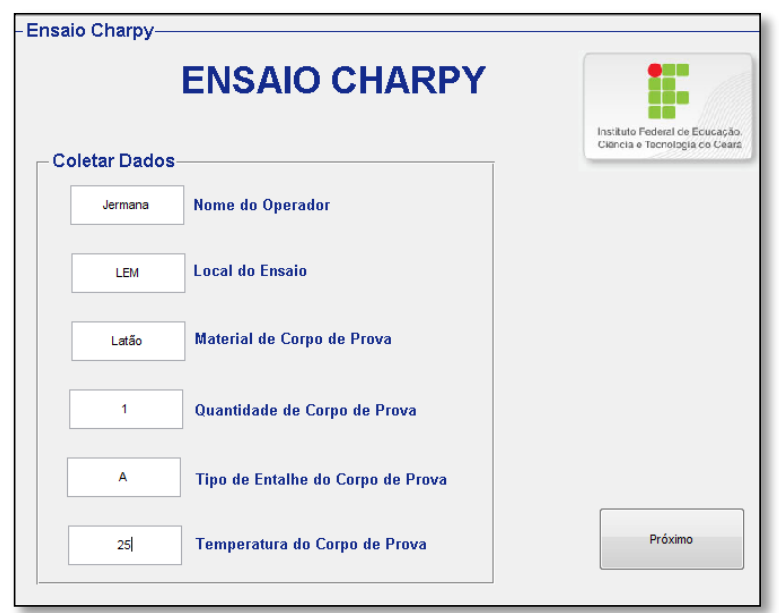

Figura 7 - Tela inicial do ensaio de impacto Charpy - Latão. 
Na Figura 13 é apresentada a segunda tela da interface gráfica. Nestas telas é possível iniciar o ensaio de impacto Charpy com a liberação do pêndulo. Como também nesta tela estão disponíveis os resultados finais do ensaio. Com os resultados apresentados nestas telas é possível realizar um estudo comparativo a cerca de um material, como também determinar as características mecânicas de um determinado corpo de prova.

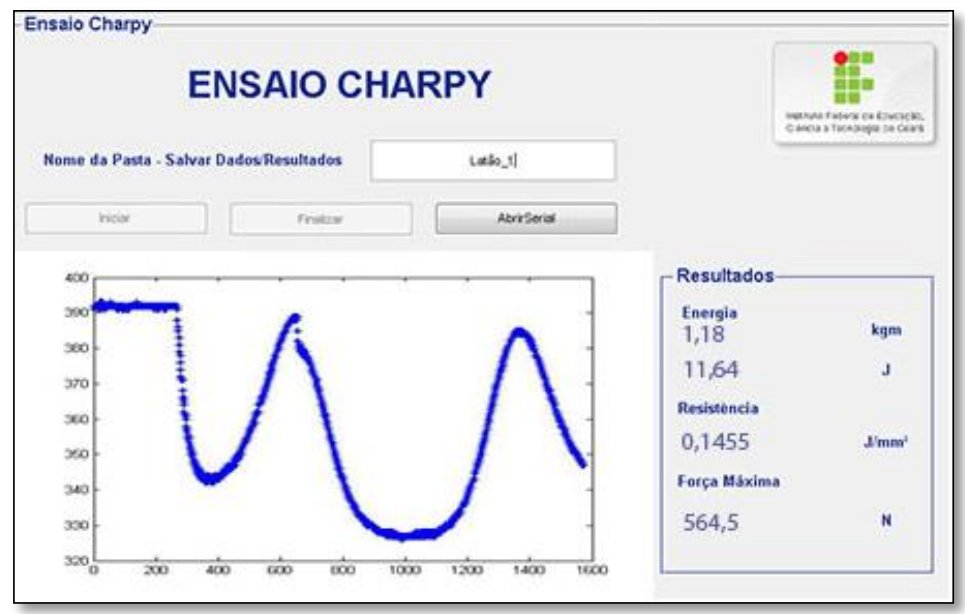

Figura 13 - Tela dos resultados do ensaio de impacto Charpy - Latão.

Os resultados apresentados nestas telas são a energia em kpm e Joules, a resistência e a força máxima de impacto, como também são disponibilizados os gráficos de deslocamento do eixo $x$ durante a realização do ensaio e o gráfico de força ao longo do tempo durante a realização do ensaio.

Após finalizar o ensaio de impacto através da interface gráfica será gerado o gráfico de força e tempo. Desta forma, torna-se possível analisar o comportamento da força ao longo do tempo durante a realização do ensaio de impacto Charpy. Na Figura 14 apresenta-se o gráfico de força ao longo do tempo do Latão.

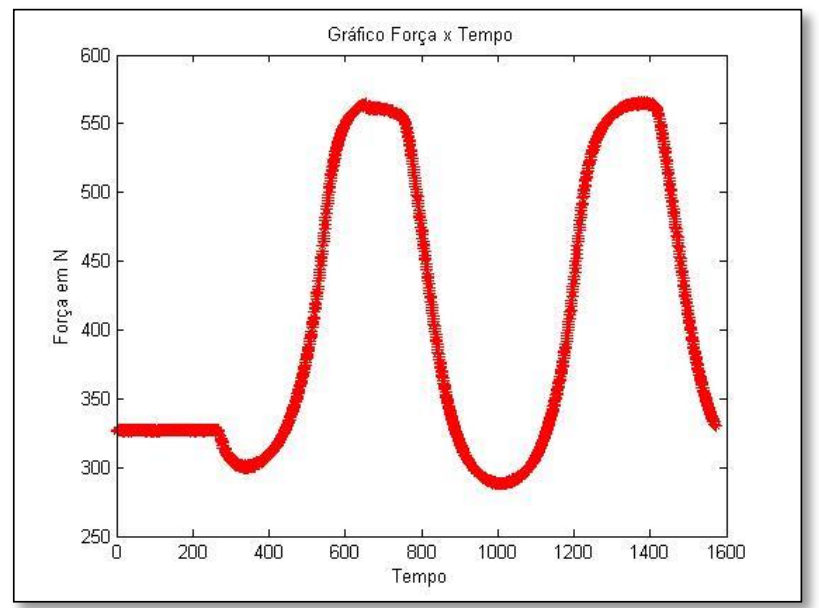

Figura 14 - Gráfico força x tempo - Latão. 
Analisando a Figura 14 pode-se determinar a força máxima de impacto que corresponde ao instante que pêndulo rompe o corpo de prova. Após o término do ensaio de impacto Charpy, todos os dados e resultados serão registrados em um arquivo txt definido pelo próprio usuário da interface gráfica. Os dados e resultados salvos em arquixo txt com o nome do arquivo especificado pelo usuário final são apresentados na Figura 15.

\begin{tabular}{|l|}
\hline Latão_1 - Bloco de notas \\
\hline Arquivo Editar Formatar Exibir Ajuda \\
\hline Nome: Jermana \\
Loca1: Lem \\
Materia1: Latão \\
Quantidade: 1 \\
Tipo de Enta1he: A \\
Temperatura: 25 \\
Energia de Impacto: $11,64 \mathrm{~J}$ \\
Energia de Impacto: $1,18 \mathrm{Kpm}$ \\
Resistencia: 0,1455 $\mathrm{J} / \mathrm{mm}^{2}$ \\
Força Máxima: 564,5 \\
\end{tabular}

Figura 15 - Dados e resultados - Latão.

A Tabela 2 apresenta a comparação dos resultados do ensaio de impacto Charpy convencional e o ensaio Automatizado. Os resultados apresentados na Tabela 2 correspondem a uma média de 10 ensaios para cada tipo de corpo de prova diferente.

Tabela 2 - Resultados dos testes finais.

\begin{tabular}{|l|c|c|c|c|}
\hline \multicolumn{1}{|c|}{$\begin{array}{c}\text { Resultados do Ensaio } \\
\text { Charpy }\end{array}$} & $\begin{array}{c}\text { Ensaio } \\
\text { Convencional Latão }\end{array}$ & $\begin{array}{c}\text { Ensaio } \\
\text { Automatizado } \\
\text { Latão }\end{array}$ & $\begin{array}{c}\text { Ensaio } \\
\text { Convencional Aço } \\
\mathbf{1 0 2 0}\end{array}$ & $\begin{array}{c}\text { Ensaio } \\
\text { Automatizado } \\
\text { Aço } 1020\end{array}$ \\
\hline $\begin{array}{l}\text { Energia de Impacto em } \\
\text { Joules }\end{array}$ & $12,64 \mathrm{~J}$ & $11,64 \mathrm{~J}$ & $14 \mathrm{~J}$ & $15 \mathrm{~J}$ \\
\hline Resistência & - & $0,1455 \mathrm{~J} / \mathrm{mm}^{2}$ & - & $0,1875 \mathrm{~J} / \mathrm{mm}^{\mathbf{2}}$ \\
\hline Força de Impacto em N & - & $564,5 \mathrm{~N}$ & - & $565,2 \mathrm{~N}$ \\
\hline $\begin{array}{l}\text { Gráfico Aceleração } x \\
\text { Tempo }\end{array}$ & Não & $\operatorname{Sim}$ & Não & Sim \\
\hline Gráfico Força x Tempo & Não & Sim & Não & Sim \\
\hline Registrar Resultados & Não & Sim & Não & Sim \\
\hline
\end{tabular}

Pela Tabela 2, pode-se analisar que os resultados de energia de impacto do ensaio automatizado são muito próximos dos resultados dos ensaios convencionais. Tendo em vista, a imprecisão de leitura de casas decimais do mostrador analógico, não se pode garanti a precisão dos resultados obtidos com o ensaio convencional. 


\section{CONSIDERAÇÕES FINAIS}

Neste trabalho, descreveram-se as etapas para automação da máquina de impacto Charpy com a utilização do Arduino e Acelerômetro para a leitura e interpretação do movimento do pêndulo Charpy através das modificações dos eixos de aceleração e, desta forma, determinar digitalmente os resultados apresentados pelo mostrador analógico da máquina de impacto, como também ampliar o número de informações coletadas no ensaio para melhoria do estudo das características mecânicas do material ensaiado.

Para comprovação da eficácia do sistema de automação da máquina de ensaio Charpy, foram realizados vários ensaios com cargas diferentes e todos os resultados foram coletados para análise e estudo.

Percebe-se que a correta instrumentação da máquina de impacto Charpy e a automação do processo permite um aumento do número de informações coletadas no ensaio, elevando consideravelmente a precisão, a confiabilidade e a segurança dos resultados obtidos.

Com o processo automatizado, a captura dos dados pode ser utilizada como base para outras pesquisas de iniciação científica, mestrado ou doutorado e mesmo auxiliando na execução de trabalhos de extensão para os setores produtivos.

\section{REFERÊNCIAS BIBLIOGRÁFICAS}

1. CALLISTER, W. Ciência e Engenharia de Materiais: Uma Introdução. Livro 50 Edição, 2008.

2. COLPAERT, H. Metalografia dos Produtos Siderúrgicos Comuns. 4ํㅡㄹ Edição. São Paulo: Edgard Blucher, 2008.

3. COSTA, F. Ensaios Tecnológicos - 3o Ciclo de Técnico em Mecânica. São Paulo, 2000.

4. DALCIN, G. Ensaio dos Materiais. Trabalho De Conclusão de Curso Apresentado ao Departamento do Curso de Engenharia Industrial Mecânica da Universidade Regional Integrada do Alto Uruguai e das Missões, 2007.

5. FIGUEIREDO, K. Aplicação de Ensaio de Impacto Charpy Instrumentado no Estudo da Tenacidade à Fratura Dinâmica nas Soldas a Arco Submerso em Aços para Caldeiras. São Carlos, 2004.

6. FREESCALE SEMICINDUCTOR. Datasheet MMA7260. EUA, 2005.

7. GOMES, E.; TAVARES, L. Uma Solução Com Arduino Para Controlar E Monitorar Processos Industriais. Trabalho de Conclusão de Curso Apresentado ao Instituto Nacional de Telecomunicações, 2013.

8. GUIMARÃES, 2010; BEPPU, M. Apostila Arduino. Rio de Janeiro, 2010.

9. JUSTEN, Alvaro. Curso de Arduino.Rio de Janeiro, 2009.

10. LUCENA, E.; MARTINS, S. Instrumentação De Um Pêndulo De Impacto Charpy. Congresso Brasileiro de Engenharia e Ciência dos Materiais, Foz do Iguaçu, 2006.

11. MCROBERTS, M. Arduino Básico. Editora Novatec, 2011.

12. MORAES, J. Instrumentação da Máquina de Ensaio Charpy. IV Simpósio de Inovação Tecnológica, 2010. 
13. SANTOS, V.M. Instrumentação e Medida, Transdução e Condicionamento Elétrico. Universidade de Aveiro, 2003.

14. SOUZA, S. Ensaios Mecânicos de Materiais: Fundamentos e Aplicações. 5 Edição, 1982.

15. THOMAZINI, D.; ALBUQUERQUE, P. Sensores Industriais: Fundamentos e Aplicações. Editora Érica, 2011.

16. TÓTH, L.; ROSSMANITH, H. P.; SIEWERT, T. A.; Historical background and development of the charpy test. Elsevier Science Ltda. and ESIS, 2002.

17. ZWINGEL, G. Instruções de Serviço para o Mecanismo Pendula para o Ensaio de Resistência ao Impacto. Leipzig, 1968. 